\title{
Validation of the diagnostic utility of urinary midkine for the detection of bladder cancer
}

\author{
DANA VU VAN, ULRIKE HEBERLING, MANFRED P. WIRTH and SUSANNE FUESSEL \\ Department of Urology, Technische Universität Dresden, D-01307 Dresden, Germany
}

Received January 12, 2016; Accepted July 12, 2016

DOI: $10.3892 / \mathrm{ol} .2016 .5040$

\begin{abstract}
As it has been demonstrated previously that midkine (also known as neurite growth-promoting factor 2) protein levels in urine of bladder cancer $(\mathrm{BCa})$ patients are increased compared to healthy controls, the present study validated the diagnostic utility of midkine in an independent patient cohort and compared the observed values with voided urine cytology (VUC), which is the current reference standard for non-invasive diagnosis of $\mathrm{BCa}$. Voided urine samples were prospectively collected from 92 BCa patients and 70 control subjects. Protein levels of midkine were assessed using a commercially available enzyme-linked immunosorbent assay and normalized to urinary creatinine. The diagnostic performance of urinary midkine was evaluated by receiver operating characteristic curves. The best combinations of sensitivities and specificities were determined by Youden's Index. Midkine concentrations were significantly elevated in urine samples from $\mathrm{BCa}$ patients compared to controls ( $\mathrm{P}<0.001$; Mann-Whitney U Test). The level of midkine was associated with disease progression, with the highest concentrations in urine specimens of patients with pT1 and $\geq$ pT2a, as well as high-grade tumors $(\mathrm{P}<0.001$; Mann-Whitney U test). Sensitivities of urinary midkine and VUC were 69.7 and $87.6 \%$, respectively. The corresponding specificities for midkine and VUC were 77.9 and $87.7 \%$, respectively. The combined use of VUC and midkine improved the sensitivity to $93.3 \%$, but reduced the specificity to $66.2 \%$. Despite its reduced discriminatory power for low-grade and low-stage $\mathrm{BCa}$, urinary midkine can be utilized for the identification of high-grade $\mathrm{pT} 1$ and $\geq \mathrm{pT} 2 \mathrm{a}$ tumors. This means that midkine may potentially be suitable for the identification of patients with high risk $\mathrm{BCa}$.
\end{abstract}

Correspondence to: Dr Susanne Fuessel, Department of Urology, Technische Universität Dresden, Fetscherstrasse 74, D-01307 Dresden, Germany

E-mail: susanne.fuessel@uniklinikum-dresden.de

Key words: enzyme-linked immunosorbent assay, neurite growth-promoting factor 2, urine, urothelial carcinoma, tumor marker

\section{Introduction}

Bladder cancer (BCa) is the most common malignancy of the urinary tract in the elderly population, and the sixth most common cancer in men worldwide (1). More than $75 \%$ of all newly diagnosed BCa cases are non-muscle-invasive lesions and $\sim 70 \%$ of patients present with pTa, $20 \%$ with pT1 and $10 \%$ with carcinoma in situ (pTIS) lesions (2,3). Although these $\mathrm{BCa}$ tumors can be resected completely by transurethral resection of the bladder (TURB), patients harbor a significant risk of tumor recurrence and progression (3). Consequently, lifelong and frequent follow-up and tumor surveillance are necessary, making $\mathrm{BCa}$ one of the most expensive diseases worldwide (4). Considering the current European Association of Urology guidelines on non-muscle-invasive bladder carcinoma (NMIBC), cystoscopy in combination with voided urine cytology (VUC) and histological evaluation of multiple bladder biopsies is the only reliable method for the diagnosis of $\mathrm{BCa}$ to date (2). In contrast to cystoscopy, VUC is relatively inexpensive. It demonstrates high sensitivity for high-grade/G3 tumors, however, due to its low sensitivity for well-differentiated, low-grade/G1 tumors, VUC may only be used as an adjunct to cystoscopy rather than replacing it (5). An additional drawback of VUC is its strong observer-dependence and the requirement for trained, experienced cytopathologists or urologists (6). However, to date it is the most commonly utilized non-invasive method for the detection of $\mathrm{BCa}$ in the current clinical routine. Previously, great effort was put into the investigation of putative urinary biomarkers suitable for the non-invasive diagnosis of $\mathrm{BCa}$ (7-10). The challenge is to develop an inexpensive test, which shows high sensitivity and specificity and which is objective and easy to perform. Despite typically demonstrating higher sensitivities compared to VUC, the majority of the current tests available lack high specificities (11). Consequently, although some of the investigated biomarkers are commercially available, a routine application of these tests is not recommended for the primary detection of $\mathrm{BCa}$ or for surveillance of NMIBC (2).

Midkine (also known as neurite growth-promoting factor 2) is a heparin-binding growth factor of $13 \mathrm{kDa}(12)$. As secretory protein, midkine is readily available in biofluids including blood and urine (13). Although midkine gene expression has been detected in several tissues of healthy individuals, including the gastrointestinal tract, kidney, spleen, lungs and thyroid, corresponding midkine protein expression could not 
be observed (13). By contrast, in a genome-wide search for abundantly expressed genes in cancerous vs. normal tissue, midkine was identified as the second highest expressed gene in the most common types of human cancer (14). Consistently, midkine protein was demonstrated to be highly expressed in various malignancies, including neuroblastoma, breast cancer, hepatocellular carcinoma, colorectal cancer and $\mathrm{BCa}(15,16)$. Although the expression of midkine protein in $\mathrm{BCa}$ and its correlation with a poor outcome in patients with invasive cancers has been previously reported (16), to the best of our knowledge there are only three studies reporting the measurement of midkine protein in urine specimens from BCa patients $(15,17,18)$. Only one study investigated whether there is a correlation between midkine protein concentration in urine and disease progression in terms of tumor stage and grade (17). Furthermore, the lack of comparison with VUC as a reference method for the non-invasive detection of $\mathrm{BCa}$ is a major drawback of these studies.

In the present study midkine was analyzed in an independent patient cohort and its diagnostic performance was compared with that of VUC. The present study aimed to evaluate the suitability of midkine as a non-invasive biomarker for $\mathrm{BCa}$.

\section{Materials and methods}

Study population, data and sample collection. BCa patients and control subjects were consecutively recruited by the Department of Urology, Technische Universität Dresden (Dresden, Germany) between May 2014 and May 2015. Urine specimens from $\mathrm{BCa}$ patients and control subjects were prospectively collected. The study was approved by the institutional review board of the Technische Universität Dresden. Written informed consent was obtained from all participants. Criteria for the inclusion of patients into the study were as follows: Age 45-80 years with suspicion of new-onset or recurrent $\mathrm{BCa}$ on the basis of initial findings during cystoscopy or during imaging examinations, who were planned to undergo primary TURB (Fig. 1). Individuals with suspected or confirmed renal cell carcinoma (RCC), cystic disease or nephrolithiasis served as controls. Those who were diagnosed with chronic cystitis (CC) as cause of their symptoms during TURB represented a distinct control group. Patients with other non-urological cancers, who had received earlier pelvic radiotherapy, as well as those who were diagnosed with papilloma or papillary urothelial neoplasm of low malignant potential or who underwent a second or third TURB were excluded from the study. Finally, a total of 162 individuals comprising $92 \mathrm{BCa}$ patients and 70 controls were selected for analysis. The final allocation to the patient and control groups was performed following histopathological examination of the resected bladder specimens that served as the reference standard in the present study. Only patients with histopathologically confirmed $\mathrm{BCa}$ were designated as $\mathrm{BCa}$ patients. Tumor staging was assessed according to Union for International Cancer Control Tumor-Node-Metastasis classification (19), and tumor grading was assessed according to World Health Organization (WHO) classification $(20,21)$. Tumor stage was evaluated as pTa, pTIS, pT1 and $\geq \mathrm{pT} 2 \mathrm{a}$ and tumor grade as low-grade and high-grade, as well as G1, G2 and G3. VUC was prepared for every urine specimen. The evaluation of all VUC specimens was performed by a single, experienced examiner, in accordance with the WHO classification (20). In cases of low cellularity, urinary cytology could not be evaluated, and therefore these samples were excluded from the analysis aimed at comparing VUC with urinary midkine. The data are reported according to the Standards for the Reporting of Diagnostic accuracy studies statements (22).

Processing of urine samples. A total of $50 \mathrm{ml}$ of random non-first-morning urine samples were obtained from every patient and control subject preoperatively. Urine dipstick analysis was performed immediately following the collection and evaluated using the automated Urisys 1800 urine analyzer (Roche Diagnostics Deutschland GmbH, Mannheim, Germany). A total of $10 \mathrm{ml}$ of urine was centrifuged at $380 \mathrm{x} \mathrm{g}$ for $3 \mathrm{~min}$ at $20^{\circ} \mathrm{C}$, and urine sediment was analyzed microscopically, using an Axio Lab.A1 microscope (Zeiss AG, Oberkochen, Germany) at magnification x400, for the presence of erythrocytes, leucocytes and bacteria. The remaining urine specimen was centrifuged at $1,500 \mathrm{x} g$ for $10 \mathrm{~min}$ at $4^{\circ} \mathrm{C}$. The supernatant was decanted and frozen in $1 \mathrm{ml}$ aliquots using tubes with protein low-binding capacity (Protein LoBind Tubes; Eppendorf, Hamburg, Germany) at $-80^{\circ} \mathrm{C}$ until the analysis of midkine. One aliquot of every urine sample was sent to the Institute of Clinical Chemistry and Laboratory Medicine (Technische Universität Dresden) for the quantification of urinary creatinine, which was used to normalize midkine concentrations. The remaining cell pellet was prefixed with Esposti's fixative overnight, centrifuged on glass slides at $1,000 \mathrm{x}$ g for $4 \mathrm{~min}$ at $20^{\circ} \mathrm{C}$, fixed with Cytofix $\mathrm{N}$ (Niepötter Labortechnik, Bürstadt, Germany) and stained by the Papanicolaou procedure (23).

Measurement of midkine by enzyme-linked immunosorbent assay (ELISA). Human midkine was determined in urine supernatants using the commercially available Human Midkine DuoSet ELISA (DY258; R\&D Systems, Inc., Minneapolis, MN, USA) according to the manufacturer's instructions, except for the concentrations of capture and detection antibodies, which were $3.2 \mu \mathrm{g} / \mathrm{ml}$ and $800 \mathrm{ng} / \mathrm{ml}$, respectively. The optical density (OD) was measured immediately following assay development using the Mithras LB 940 Multimode Microplate Reader (Berthold Technologies GMBH \& Co. KG, Bad Wildbad, Germany) set to $450 \mathrm{~nm}$. The OD at $570 \mathrm{~nm}$ (reference wavelength) was subtracted from the readings at $450 \mathrm{~nm}$. Samples were randomized across multiple ELISA plates and measured consecutively. A standard curve was constructed by generating a four parameter logistic curve-fit using GraphPad Prism version 6.05 for Windows (GraphPad Software Inc., La Jolla, CA, USA). The measured midkine concentrations in urine were normalized to the corresponding creatinine values and expressed as $\mu \mathrm{g}$ midkine/g creatinine.

Statistical analysis. The two-tailed Mann Whitney U nonparametric test was performed to evaluate differences in urinary midkine concentrations between BCa patients and controls, and with respect to tumor stage and grade. Determination of Spearman's rank correlation coefficient $\left(\mathrm{r}_{\mathrm{s}}\right)$ 


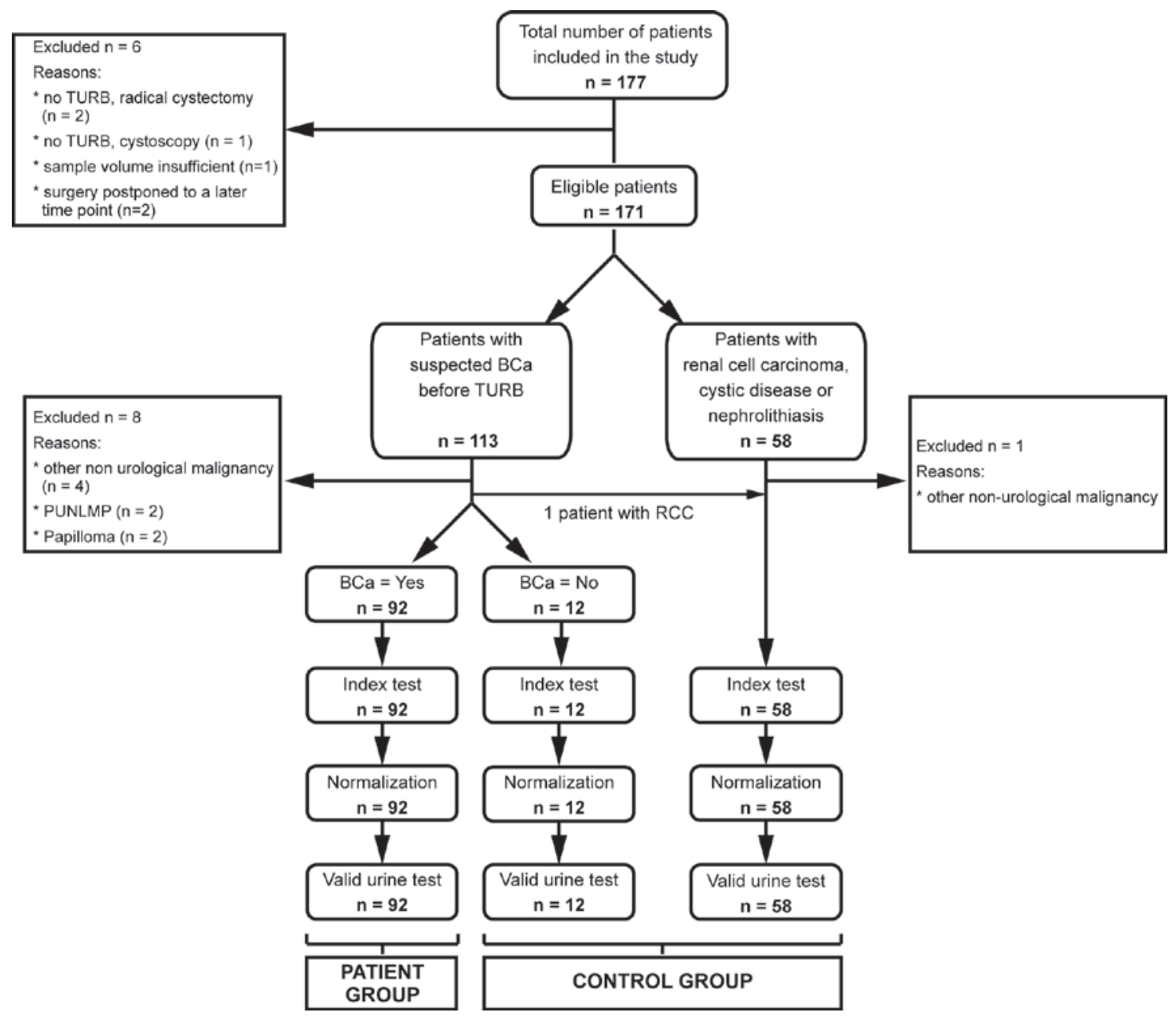

Figure 1. Flow chart showing patient recruitment and number of eligible patients. A total of 177 patients were initially recruited for the study. Due to the indicated reasons, 6 patients were subsequently excluded. Of the remaining 171 patients, 113 were suspected to have BCa and 58 patients constituted the control group. Following histopathological examination of the resected bladder tissue, an additional 9 patients were excluded. One patient initially suspected to have $\mathrm{BCa}$ was diagnosed with RCC and therefore switched to the control group. Therefore, urinary midkine was analyzed for $92 \mathrm{BCa}$ patients and 70 controls. A total of 12 patients initially suspected to have $\mathrm{BCa}$, which could not be confirmed following TURB, formed the distinct control group of chronic cystitis. PUNLMP, papillary urothelial neoplasm of low malignant potential; RCC, renal cell carcinoma; BCa, bladder cancer; TURB, transurethral resection of the bladder.

revealed potential correlations between midkine concentrations and tumor stage and grade. Statistical analyses were performed using GraphPad Prism version 6.05 for Windows. Data are plotted as box plots, where the bottom and top of the boxes represent the first and third quartiles, respectively. The median is shown as a solid line within the box, and the ends of the whiskers represent the 10-90 percentile. Data not included between the whiskers are defined as outliers and are marked as single circle symbols. The diagnostic performance of urinary midkine was determined using receiver operating characteristic (ROC) curve analyses and the corresponding area under the curve (AUC) values. ROC analyses were performed using IBM SPSS Statistics for Windows version 23.0 (IBM SPSS, Armonk, NY, USA). Youden's Index helped to calculate the best cut-off values for midkine with the best combination of sensitivity and specificity (24). For all analyses, $\mathrm{P}<0.05$ was considered to indicate a statistically significant difference. Positive predictive value (PPV), negative predictive value (NPV), positive likelihood ratio (pLR), negative likelihood ratio (nLR) and accuracy were calculated according to standard statistical methods (25).

\section{Results}

Characteristics of BCa patients and control subjects. A total of 177 patients and control subjects were recruited for participation in the present study. A total of 162 individuals were eligible for analysis (Fig. 1). Among them there were 92 patients diagnosed with $\mathrm{BCa}$ and 70 controls. The majority of control subjects suffered from RCC (48.6\%), followed by nephrolithiasis (22.9\%), CC (17.1\%) and other benign urological diseases (11.4\%). Almost $80 \%$ of the $\mathrm{BCa}$ patients were male, compared to $74 \%$ of the control subjects. The median age was 71 years (range, 50-80 years) and 64.5 years (range, $43-80$ years) in the patient group vs. the control group, respectively. There were seven times more cases of new-onset bladder tumors than recurrent tumors. The relative distributions of $\mathrm{pTa}, \mathrm{pT} 1$ and $\geq \mathrm{pT} 2 \mathrm{a}$ tumors were 50.0, 22.8 and $15.2 \%$, respectively. A single patient exhibited a tumor categorized as pTIS only, whereas 10 patients showed concomitant pTIS. According to the severity of the diagnosed tumor stage, patients with pTa and pT1 tumors and concomitant pTIS were assigned pTIS, whereas BCa patients 
Table I. Demographic, clinical and histopathological characteristics of patients with bladder carcinoma. ${ }^{a}$

\begin{tabular}{|c|c|c|}
\hline Category & $\mathrm{n}$ & $\%$ \\
\hline Total patients & 92 & 100.0 \\
\hline \multicolumn{3}{|l|}{ Gender } \\
\hline Male & 73 & 79.3 \\
\hline Female & 19 & 20.7 \\
\hline \multicolumn{3}{|l|}{ Age $^{\mathrm{b}}$, years } \\
\hline$<71$ & 44 & 47.8 \\
\hline$\geq 71$ & 48 & 52.2 \\
\hline \multicolumn{3}{|l|}{ Tumor } \\
\hline Primary & 80 & 87.0 \\
\hline Recurrent & 12 & 13.0 \\
\hline \multicolumn{3}{|c|}{ Voided urine cytology } \\
\hline Positive & 78 & 84.8 \\
\hline Negative & 11 & 12.0 \\
\hline Not evaluable & 3 & 3.2 \\
\hline \multicolumn{3}{|l|}{ Tumor stage } \\
\hline pTa & 46 & 50.0 \\
\hline pT1 & 21 & 22.8 \\
\hline pTIS & 11 & 12.0 \\
\hline pTIS only & 1 & 1.2 \\
\hline$+\mathrm{pTa}$ & 2 & 2.2 \\
\hline$+\mathrm{pT} 1$ & 4 & 4.3 \\
\hline$+\geq \mathrm{pT} 2 \mathrm{a}$ & 4 & 4.3 \\
\hline$\geq \mathrm{pT} 2 \mathrm{a}$ & 14 & 15.2 \\
\hline \multicolumn{3}{|c|}{ Concomitant $\mathrm{PCa}$} \\
\hline Yes & 9 & 9.8 \\
\hline No & 83 & 90.2 \\
\hline \multicolumn{3}{|c|}{ Tumor grade (WHO 2004) } \\
\hline Low & 17 & 18.5 \\
\hline High & 75 & 81.5 \\
\hline \multicolumn{3}{|c|}{ Tumor grade $^{c}$ (WHO 1973) } \\
\hline G1 & 16 & 17.5 \\
\hline $\mathrm{G} 2$ & 40 & 44.0 \\
\hline G3 & 35 & 38.5 \\
\hline
\end{tabular}

${ }^{\mathrm{a}} \mathrm{A}$ total of $92 \mathrm{BCa}$ patients were included in the present study. Among them were patients with primary and recurrent disease, and various tumor stages and grades. The table shows absolute and relative distribution of gender, age and clinicopathological parameters. ${ }^{b}$ Dichotomized at median. 'Tumor grade was available for only 91 patients. TIS, carcinoma in situ; PCa, prostate cancer. WHO, World Health Organization.

with muscle invasive tumors, who exhibited concomitant pTIS, were allocated to the group $\geq$ pT 2 a. A total of $9 / 92 \mathrm{BCa}$ patients $(9.8 \%)$ simultaneously exhibited prostate cancer (PCa). Furthermore, the patient cohort comprised 4.4 times more patients with high-grade tumors than low-grade tumors. In accordance to the WHO classification, these tumors were classified as $17.5 \% \mathrm{G} 1,44.0 \% \mathrm{G} 2$ and $38.5 \% \mathrm{G} 3$ (21). A
Table II. Demographic, clinical and histopathological characteristics of control subjects. ${ }^{a}$

\begin{tabular}{lcc}
\hline Category & $\mathrm{n}$ & $\%$ \\
\hline Total patients & 70 & 100.0 \\
Gender & & \\
$\quad$ Male & 52 & 74.3 \\
Female & 18 & 25.7 \\
Age, years & & \\
$\quad<64.5$ & 35 & 50.0 \\
$\geq 64.5$ & 35 & 50.0 \\
Voided urine cytology & & \\
Positive & 8 & 11.4 \\
Negative & 57 & 81.4 \\
$\quad$ Not evaluable & 5 & 7.2 \\
Diagnosis & & \\
Renal cell carcinoma & 34 & 48.6 \\
Nephrolithiasis & 16 & 22.9 \\
Chronic cystitis & 12 & 17.1 \\
Benign urological disease & 8 & 11.4 \\
\hline
\end{tabular}

${ }^{\text {a } I n}$ total, 70 subjects diagnosed with malignant and non-malignant diseases of the kidney, as well as non-malignant diseases of the bladder, were included in the control group. The table shows the absolute and relative distribution of gender, age and clinicopathological pa-

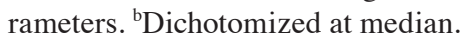

summary of all demographic, clinical and histopathological data is depicted in Tables I and II.

Utility of urinary midkine as a biomarker for the detection of $B C a$. BCa patients often simultaneously suffer from $\mathrm{PCa}$. To exclude any influence of $\mathrm{PCa}$ on urinary midkine concentration, patients with and without concomitant $\mathrm{PCa}$ were compared regarding their urinary midkine levels. No statistically significant difference was observed in the urinary midkine levels of BCa patients and those who additionally were diagnosed with $\mathrm{PCa}(\mathrm{P}=0.777$; Mann Whitney $\mathrm{U}$ test; data not shown). For this reason, patients diagnosed with $\mathrm{BCa}$ and $\mathrm{PCa}$ were not excluded from the patient group. In addition, subdividing the group of control subjects into patients with RCC, nephrolithiasis and benign urological disease also revealed no statistically significant differences in midkine concentrations (data not shown). Patients diagnosed with CC as the cause of their symptoms were initially examined separately from the remaining control subjects.

The levels of urinary midkine in the respective groups are shown in Fig. 2. The median midkine concentrations in urine specimens from patients with $\mathrm{CC}$ and $\mathrm{BCa}$ were 0.45 and $1.35 \mu \mathrm{g}$ midkine/g creatinine, respectively. Thus, urinary midkine levels were significantly elevated in patients with $\mathrm{BCa}$ compared to $\mathrm{CC}$ patients (3-fold increase; $\mathrm{P}=0.002$ ). This was also true for the comparison of patients with RCC, nephrolithiasis and other benign urological diseases with $\mathrm{BCa}$ patients (3.8-fold increase; $\mathrm{P}<0.001)$. In addition, both control subgroups (group 1, patients with RCC, nephrolithiasis and other benign urological disease; group 2, patients with chronic cystitis) were not significantly different when compared with 


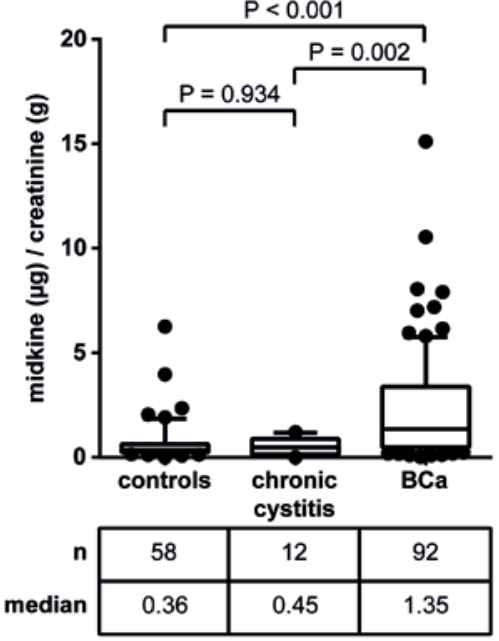

Figure 2. Urinary levels of midkine in patients with BCa compared to controls. Urine of $\mathrm{BCa}$ patients, patients with chronic cystitis and further control subjects was used for the detection of urinary midkine using a commercially available enzyme-linked immunosorbent assay. The table shows the total number of patients per group, as well as the corresponding median midkine levels. Data were normalized to urinary creatinine ( $\mu \mathrm{g} / \mathrm{g}$ creatinine). $\mathrm{P}<0.05$ was considered statistically significant (Mann Whitney U test). BCa, bladder cancer.

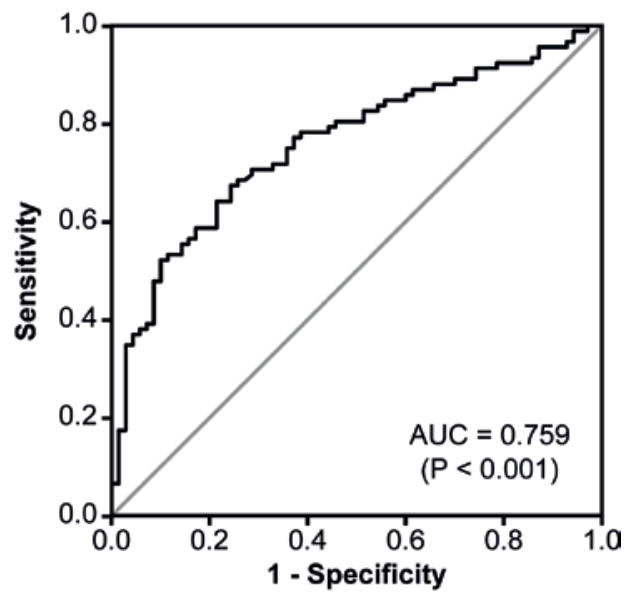

Figure 3. Receiver operating characteristic curve analysis of urinary midkine for the diagnostic discrimination of $\mathrm{BCa}$ patients and controls. The level of midkine concentration in urine specimens of $92 \mathrm{BCa}$ patients and 70 controls was determined. The cut-off value with the best combination of sensitivity $(69.7 \%)$ and specificity $(76.9 \%)$ was evaluated by Youden's Index to be $0.71 \mu \mathrm{g} / \mathrm{g}$ creatinine. The P-value tested the null hypothesis that the $\mathrm{AUC}=0.50$. If $\mathrm{P}<0.05$, the AUC was significantly different from 0.5 and the null hypothesis could be rejected. AUC, area under the curve; CI, confidence interval. BCa, bladder cancer.

each other $(\mathrm{P}=0.934)$. Consequently, patients with $\mathrm{CC}, \mathrm{RCC}$, nephrolithiasis and other benign urological diseases were considered together as a single control group for further analyses. ROC analysis for urinary midkine as a diagnostic BCa marker revealed an AUC of 0.759 (Fig. 3).

To assess potential associations of midkine with tumor stage and grade, the patient group was subdivided into pTa, pTIS, pT1 and $\geq \mathrm{pT} 2 \mathrm{a}$, as well as low-grade vs. high-grade, and midkine levels were evaluated relative to controls (Fig. 4). Urinary midkine levels were significantly elevated in all four patient groups relative to controls, however, the more

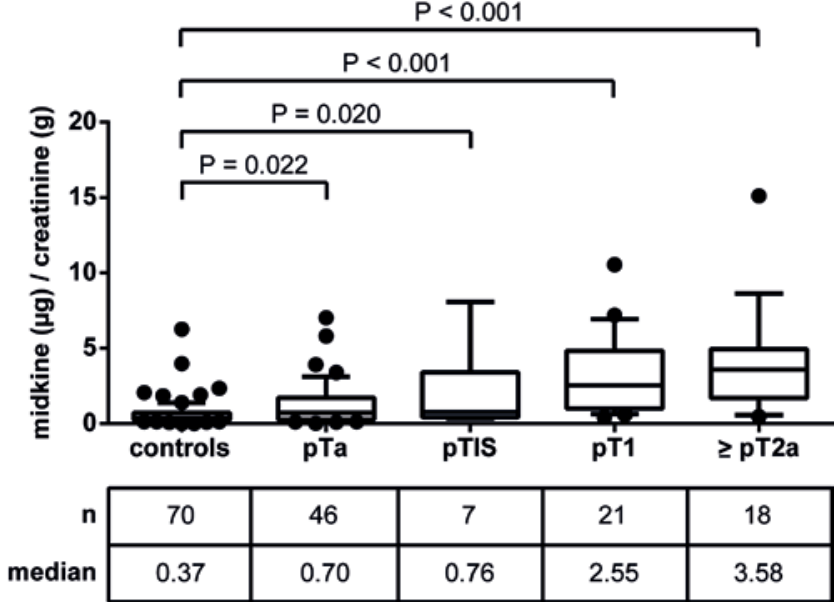

Figure 4. Correlation of urinary midkine level with tumor stage. Following the histopathological examination of the resected tumor tissue, bladder cancer patients were classified regarding their tumor stage as pTa, pTIS, pT1 or $\geq$ pT2a. Patients with pTIS only, as well as patients with tumors that showed concomitant pTIS (with the exception of $\geq p T 2 a / p T I S)$, formed the pTIS group. The control group included patients with chronic cystitis, renal cell carcinoma, cystic disease and nephrolithiasis. The table shows the total number of patients per group as well as the corresponding median midkine levels. Data were normalized to urinary creatinine $(\mu \mathrm{g} / \mathrm{g}$ creatinine). $\mathrm{P}<0.05$ was considered statistically significant (Mann Whitney U test). TIS, carcinoma in situ.

progressed the tumor, the higher the median midkine concentration. Therefore, patients with pT1 and muscle-invasive $\geq$ pT2a tumors were more distinguishable from controls compared with patients with pTa and pTIS tumors. Spearman's rank correlation coefficient was $0.536(\mathrm{P}<0.001)$, reflecting a moderate positive, but highly significant correlation between midkine levels and tumor stage.

A similar observation was made with regard to tumor grade (Fig. 5). Midkine concentrations in patients with low-grade tumors were not significantly different from controls $(\mathrm{P}=0.818)$, whereas high-grade tumors demonstrated significantly elevated urinary midkine levels $(\mathrm{P}<0.001$; Fig. 5A). Using the WHO classification, in the present patient cohort almost all patients with low-grade tumors corresponded to G1 tumors, whereas the high-grade group included patients with G2 and G3 tumors (21). Patients with G2 and G3 tumors showed significantly increased urinary midkine concentrations compared to controls $(\mathrm{P}<0.001)$, whereas patients with G1 tumors did not $(\mathrm{P}=0.720$; Fig. $5 \mathrm{~B})$. The calculation of Spearman's rank correlation coefficient revealed a moderate positive, highly significant correlation $\left(\mathrm{r}_{\mathrm{s}}=0.453 ; \mathrm{P}<0.001\right)$.

Comparison of diagnostic performance of urinary midkine and VUC. As VUC is the reference standard for non-invasive detection of $\mathrm{BCa}$, the present study aimed to compare the diagnostic power of VUC with that of urinary midkine. For this comparison, only urine specimens that exhibited a valid urine test for midkine and an evaluable VUC were included. This was true for 154/162 urine specimens. ROC curve analyses helped to assess the diagnostic performance of urinary midkine for the discrimination of $\mathrm{BCa}$ patients and controls, and to calculate the best cut-off value. Using a cut-off concentration of $0.71 \mu \mathrm{g}$ midkine/g creatinine the 


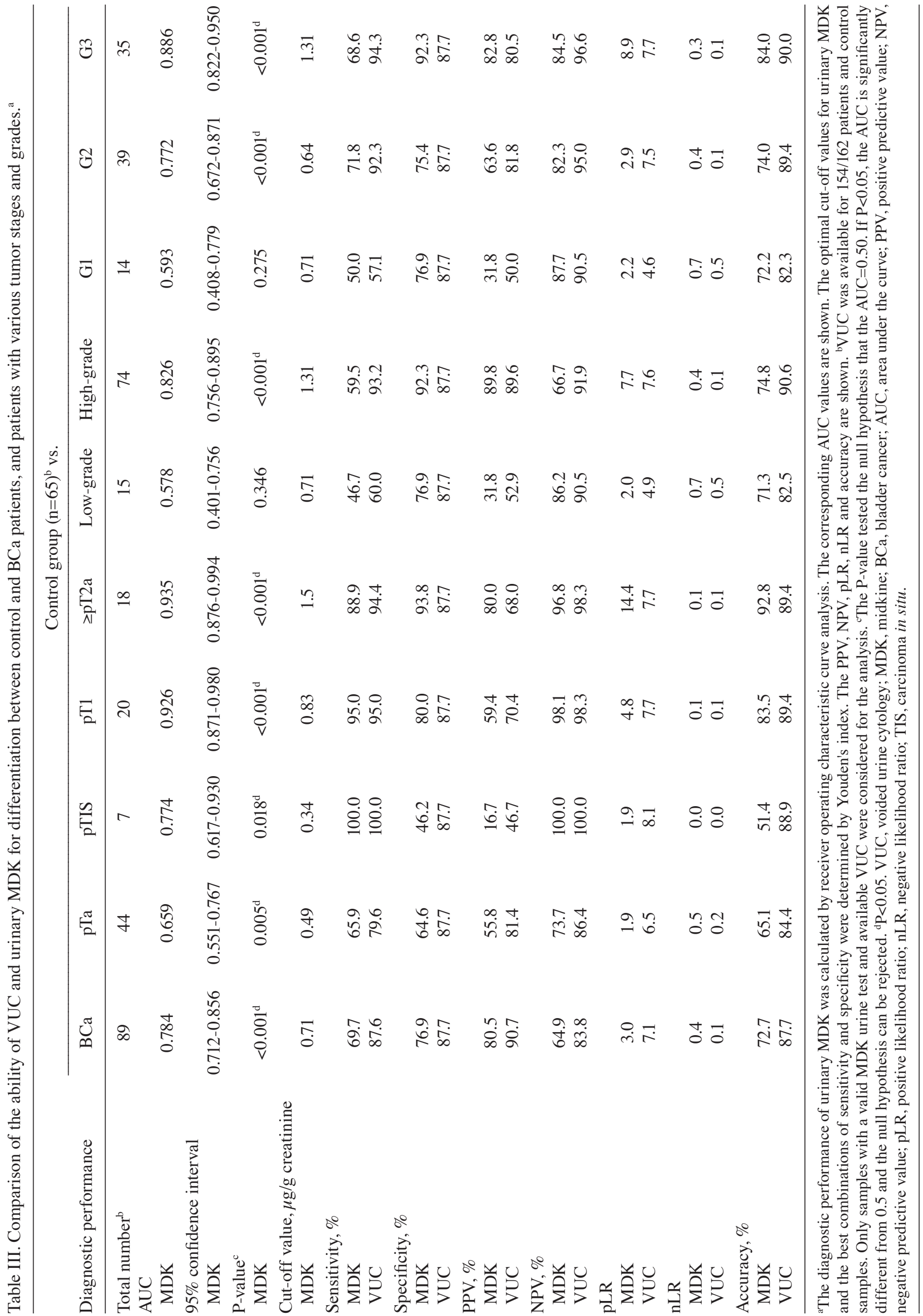


Table IV. Combinatory utility of MDK and VUC for the diagnosis of bladder cancer. ${ }^{\text {a }}$

\begin{tabular}{lcccc}
\hline Diagnostic performance & VUC only $^{\mathrm{b}}$ & MDK only $^{\mathrm{c}}$ & VUC and MDK $^{\mathrm{d}}$ & VUC and/or MDK $^{\mathrm{e}}$ \\
\hline Sensitivity, \% & 87.6 & 69.7 & 64.0 & 93.3 \\
Specificity, \% & 87.7 & 77.9 & 98.5 & 66.2 \\
PPV, \% & 90.7 & 80.5 & 98.3 & 79.0 \\
NPV, $\%$ & 83.8 & 64.9 & 66.7 & 87.8 \\
pLR & 7.1 & 3.0 & 41.6 & 2.8 \\
nLR & 0.1 & 0.4 & 0.4 & 0.1 \\
Accuracy, $\%$ & 87.7 & 72.7 & 78.6 & 81.8 \\
\hline
\end{tabular}

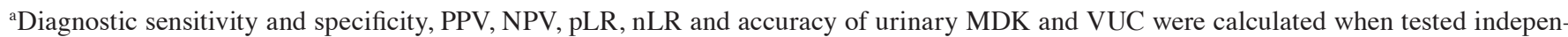
dently or in combination. Patients with a suspicious VUC in combination with urine MDK $>$ the cut-off value of $0.71 \mu \mathrm{g} / \mathrm{g}$ creatinine (variant 1 ) or patients with a suspicious VUC and/or urine MDK > the cut-off value (variant 2) were allocated to show a positive test result. Histopathological examination of the resected tumor tissue served as a reference standard to define the disease status of the patient. ${ }^{b} \mathrm{VUC}$ was available for only 154 patients and control samples ${ }^{\mathrm{c}} \mathrm{Cut}$-off value of urine midkine, $0.71 \mu \mathrm{g} / \mathrm{g}$ creatinine; ${ }^{\mathrm{d}}$ Variant 1 , VUC positive and urine midkine $>$ the cut-off value; ${ }^{e}$ Variant 2, VUC positive and/or urine midkine > the cut-off value. MDK, midkine; VUC, voided urine cytology; PPV, positive predictive value; NPV, negative predictive value; $\mathrm{pLR}$, positive likelihood ratio; $\mathrm{nLR}$, negative likelihood ratio.

A

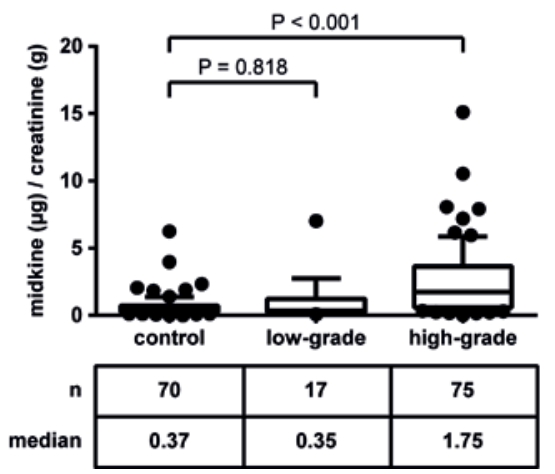

B

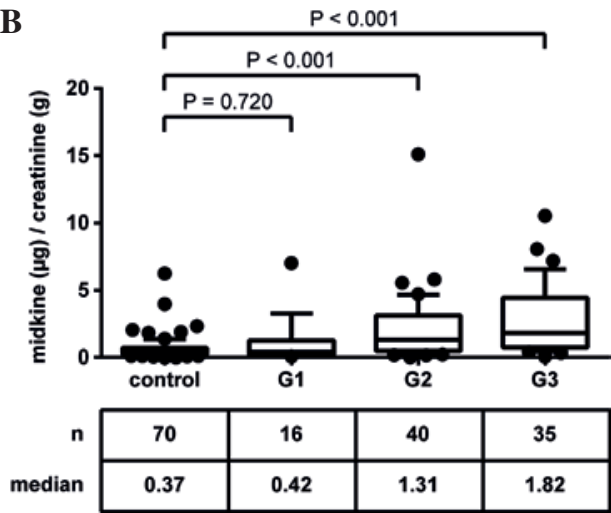

Figure 5. Correlation of urinary midkine levels with tumor grade. Following the histopathological examination of the resected tumor tissue, bladder cancer patients were classified regarding their tumor grade in (A) low-grade and high-grade (WHO 2004), and (B) G1, G2, G3 (WHO 1973, available only for 91 patients). The control group included patients with chronic cystitis, renal cell carcinoma, cystic disease and nephrolithiasis. The tables show the total number of patients per group, as well as the corresponding median midkine level. Data were normalized to urinary creatinine $(\mu \mathrm{g} / \mathrm{g}$ creatinine $)$. $\mathrm{P}<0.05 \mathrm{was}$ considered statistically significant (Mann Whitney U test). WHO, World Health Organization.

AUC was 0.784 and the sensitivity and specificity of urinary midkine were 69.7 and $76.9 \%$, respectively. VUC clearly outperformed urinary midkine determination with $87.6 \%$ sensitivity and $87.7 \%$ specificity (Table III). In addition, VUC more convincingly detected the disease than urinary midkine with positive and negative likelihood ratios of 7.1 and 0.1 (VUC) and 3.0 and 0.4 (urinary midkine), respectively. In the next step, the capability of VUC and urinary midkine to discriminate patients with various tumor stages and grades from controls was assessed. AUC values were determined for pTa, pTIS, pT1 and $\geq$ pT 2 a to be $0.659,0.774$, 0.926 and 0.935 , respectively. The lowest diagnostic performance for the selected cut-off values was observed for pTa tumors with a sensitivity of $65.9 \%$, followed by pTIS tumors (100.0\%), pT1 tumors $(95.0 \%)$ and $\geq$ pT2a tumors $(88.9 \%)$. The corresponding specificities were $64.6 \%$ (pTa), $46.2 \%$ (pTIS), $80.0 \%$ (pT1) and $93.8 \%$ ( $\geq \mathrm{pT} 2 \mathrm{a}$ ). The best discriminatory power of the test could be reached for patients diagnosed with pT1 and $\geq$ pT 2 a tumors, whereas the identification of less progressed pTa tumors was diminished. In contrast, VUC achieved similar sensitivities (pTa, 79.6\%; pTIS,
100.0\%; pT1, 95.0\%; >pT2a, 94.4\%) but higher specificities (87.7\% each) compared to midkine. Regarding tumor grade, low-grade tumors could not be discriminated from controls by VUC (sensitivity, $60.0 \%$; specificity, $87.7 \%$ ) or urinary midkine (AUC, 0.578; sensitivity: 46.7\%; specificity, 76.9\%). By contrast, high-grade tumors were highly distinguishable by VUC (sensitivity, 93.2\%; specificity, 87.7\%) and urinary midkine (AUC, 0.826; sensitivity, 59.5\%; specificity, 92.3\%; Table III).

Combinatory ability of urinary midkine and VUC to detect $B C a$. To investigate whether the combination of urinary midkine and VUC had a synergistic effect on the detection of $\mathrm{BCa}$, diagnostic sensitivity and specificity, PPV and NPV, pLR and $\mathrm{nLR}$, as well as accuracy of urinary midkine and VUC were calculated when tested independently or in combination (Table IV). Patients with a positive VUC in combination with urinary midkine above the cut-off value of $0.71 \mu \mathrm{g} / \mathrm{g}$ creatinine (variant 1) or patients with a positive VUC and/or urinary midkine above the cut-off value (variant 2) were considered BCa positive. 
The addition of midkine to VUC on the basis of variant 1 lowered the sensitivity considerably, from $87.6 \%$ (VUC) and $69.7 \%$ (midkine) to $64.0 \%$, but increased the specificity from $87.7 \%$ (VUC) and $77.9 \%$ (midkine) to $98.5 \%$. By contrast, the combination of VUC and midkine according to variant 2 improved the sensitivity to $93.3 \%$, but reduced the specificity to $66.2 \%$. Therefore, urinary midkine was not able to complement VUC or replace it as a non-invasive method to detect $\mathrm{BCa}$ in the present study.

\section{Discussion}

The aim of the present study was to validate urinary midkine protein, suggested as a convenient biomarker for the non-invasive detection of $\mathrm{BCa}(17,18)$, in an independent patient cohort and to compare its diagnostic utility with that of VUC.

With 3.8 times more male than female subjects and a median age of 71 years, the present patient cohort reflected the stated epidemiological parameters for BCa in Europe (2). In addition, $>80 \%$ of patients with new-onset and recurrent $\mathrm{BCa}$ exhibited NMIBC, which is in line with the observations of Babjuk et al (2). By the inclusion of patients with RCC, nephrolithiasis and non-malignant urological diseases (of the bladder or kidney) in the control group, the present study was able to investigate potential impacts of these conditions on the determination of urinary midkine levels. This is an important issue, as it was reported that expression of midkine protein may only be detected in the kidney of adults at very low levels (12). Furthermore, a potential influence of renal function on the performance of urine-based markers to detect BCa was demonstrated (26). A major advantage of the present study in contrast to existing reports is that the recruited control subjects appear more suitable than healthy individuals for evaluation of putative urinary biomarkers, as they more closely reflect the target population and permit the definition of better cut-off values for the tested parameters. In particular, the $\mathrm{CC}$ group is of great value, as these patients resemble the $\mathrm{BCa}$ patients most closely with regard to clinical symptoms. Using healthy controls with no evidence of urological disease may lead to overestimation of the obtained results, as the calculated threshold value may be too low.

The analysis of urinary midkine protein revealed significantly elevated midkine concentrations in BCa patients vs. controls. This observation is in agreement with two previous reports that also described increased midkine protein levels in urine specimens of $\mathrm{BCa}$ patients $(17,18)$. In the present study, the calculated AUC value of urinary midkine for the prediction of $\mathrm{BCa}$ was 0.759 , which was lower compared to the AUC value of 0.857 for midkine reported by Soukup et al (18). This may be due to the differing composition of the control group used by Soukup et al (18), who compared patients with primary occurrence of $\mathrm{BCa}$ with healthy controls. Due to the aforementioned reasons, using healthy controls may lead to an overestimation of the obtained differences between $\mathrm{BCa}$ patients and controls. Although overexpression of midkine protein has been reported for cancerous prostate tissue (27), the comparison of midkine concentrations in urine specimens from $\mathrm{BCa}$ patients with concomitant $\mathrm{PCa}$ revealed no statistically significant difference compared with BCa patients without PCa (data not shown). Contrasting with survivin, one of the most promising biomarkers in the diagnosis of $\mathrm{BCa}(28)$, $\mathrm{PCa}$ does not contribute to urinary midkine concentrations in the present study.

Additional evaluation of midkine protein levels in urine specimens of BCa patients with various tumor stages and grades revealed a correlation between urinary midkine concentration and disease severity. This result is in agreement with other molecular-based tests, aimed at the quantification of soluble proteins associated with $\mathrm{BCa}$, for example, the analysis of vascular endothelial growth factor or bladder tumor antigen in urine (29-31). The more progressed the tumor was, the higher the median urinary midkine concentration. Despite significantly elevated midkine levels in the urine of $\mathrm{BCa}$ patients with pTa tumors compared to controls, the diagnostic performance of midkine for the identification of these patients was low (AUC, 0.659). By contrast, pT1 and $\geq \mathrm{pT} 2$ a tumors were highly distinguishable from controls (AUC, 0.926 and 0.935 , respectively), however, a further discrimination of pT1 and muscle-invasive bladder tumors using midkine failed. In addition, urinary midkine levels of patients with low-grade tumors were not significantly different from controls, whereas patients with high-grade tumors exhibited significantly elevated urinary midkine levels. These observations are in accordance with Shimwell et al (17), who reported substantial elevation of midkine protein in the urine of BCa patients, although not in those with early-stage, low-grade disease. Notably, these and the present results may reflect the transcript level (17), as it has been demonstrated that urinary midkine mRNA ia more sensitive in the detection of high-grade and high-stage $\mathrm{BCa}$ (32). In addition, a number of other solid tumors express midkine at high levels, and its expression increases with advancing tumor stage $(15,33)$. The low benefit of midkine as a diagnostic marker for low-grade pTa and pTIS tumors is unfortunate but comparable to other urine-based protein markers, including cytokeratin 18 (34) and survivin (35). However, as 70\% of patients with NMIBC have pTa tumors and recurrence is the main problem in these patients (3), urinary midkine alone is not reliable as a diagnostic marker for the surveillance of patients with a history of $\mathrm{BCa}$. Nevertheless, midkine was observed to be highly suitable for the identification of more severe pT1 and $\geq \mathrm{pT} 2 \mathrm{a}$ lesions. This may offer the opportunity to use midkine as a marker for the identification of high-risk patients.

The comparison of urinary midkine with VUC as the current reference standard for non-invasive diagnosis of $\mathrm{BCa}$ may assist with evaluation of this biomarker. VUC clearly outperformed urinary midkine, with an overall sensitivity and specificity of 87.6 and $87.7 \%$ compared with 69.7 and $77.9 \%$, respectively. Comparable to Soukup et al (18), but in contrast to further reports, which claimed sensitivities of $\sim 35 \%$ for VUC (8,36-38), the sensitivity of VUC was markedly high in the present study, at $>87 \%$. This observation most probably resulted from the 4.4-fold higher number of high-grade tumors compared to low-grade tumors in the present patient cohort, as it is well known that VUC is most suitable for the diagnosis of high-grade tumors (39-41). Taking this into account, the sensitivity of VUC for BCa patients with low-grade and G1 tumors was only 60.0 and $57.1 \%$, respectively. However, with sensitivities of $46.7 \%$ (low-grade tumors) and $50.0 \%$ (G1 tumors), urinary midkine was an even poorer detection method. The comparison of midkine with other biomarker studies is limited 
by variations in study design, composition of investigated patient and control cohorts or applied analysis methods. Nevertheless, contrasting with urinary midkine, survivin was more sensitive and less specific than urine cytology according to previously published data $(35,42-44)$.

A newly developed urinary test must achieve sensitivities of 90-95\% to earn a broad acceptance as an alternative to cystoscopy $(45,46)$. The challenge is to identify adjunct biomarkers that assist with increasing the low sensitivity of VUC while maintaining its high specificity. Previously, the concept of a single marker for the diagnosis of $\mathrm{BCa}$ was replaced by the idea to establish multi-marker panels that facilitate higher sensitivities $(18,47,48)$. However, with the combined use of VUC and urinary midkine, the present study was not able to improve the diagnostic performance compared with VUC alone. An increased combined sensitivity was accompanied by a reduced combined specificity. Similar observations were made by several other groups, who showed that the more markers used in parallel for the detection of $\mathrm{BCa}$ the lower the specificity of the test $(18,49)$. Therefore, the approach suggested by Lalkhen and McCluskey may serve as good alternative (25). Patients who are initially positive to a test with high sensitivity/low specificity should be subjected to a second test with low sensitivity/high specificity. They proposed that in this way, almost all of the false positives may be correctly identified as disease negative. Although the utility of urinary midkine protein for the early, non-invasive diagnosis of low-grade, less progressed bladder tumors is limited, urinary midkine was observed to be a suitable marker for the identification of patients at high risk of tumor progression. In addition, in combination with additional transcriptional or proteomic markers (e.g. keratin 20, hyaluronic acid/hyaluronidase), that will recognize low-grade tumors more effectively $(50,51)$, midkine may have the potential to contribute to the non-invasive diagnosis and monitoring of BCa.

\section{Acknowledgements}

The present study was funded by the German Federal Ministry of Education and Research (grant no., 16SV6070).

\section{References}

1. Torre LA, Bray F, Siegel RL, Ferlay J, Lortet-Tieulent J and Jemal A: Global cancer statistics, 2012. CA Cancer J Clin 65: 87-108, 2015

2. Babjuk M, Burger M, Zigeuner R, Shariat SF, van Rhijn BW, Compérat E, Sylvester RJ, Kaasinen E, Böhle A, Palou Redorta J and Rouprêt M; European Association of Urology: EAU guidelines on non-muscle-invasive urothelial carcinoma of the bladder: Update 2013. Eur Urol 64: 639-653, 2013.

3. van Rhijn BWG, Burger M, Lotan Y, Solsona E, Stief CG, Sylvester RJ, Witjes JA and Zlotta AR: Recurrence and Progression of disease in non-muscle-invasive bladder cancer: From epidemiology to treatment strategy. Eur Urol 56: 430-442, 2009.

4. Svatek RS, Hollenbeck BK, Holmäng S, Lee R, Kim SP, Stenzl A and Lotan Y: The economics of bladder cancer: Costs and considerations of caring for this disease. Eur Urol 66: 253-262, 2014.

5. Talwar R, Sinha T, Karan SC, Doddamani D, Sandhu A Sethi GS, Srivastava A, Narang V, Agarwal A and Adhlakha N: Voided urinary cytology in bladder cancer: Is it time to review the indications? Urology 70: 267-271, 2007.

6. Raitanen MP, Aine R, Rintala E, Kallio J, Rajala P, Juusela H and Tammela TL; FinnBladder Group: Differences between local and review urinary cytology in diagnosis of bladder cancer. An interobserver multicenter analysis. Eur Urol 41: 284-289, 2002.
7. Lokeshwar VB, Habuchi T, Grossman HB, Murphy WM, Hautmann SH, Hemstreet GP III, Bono AV, Getzenberg RH, Goebell P, Schmitz-Dräger BJ, et al: Bladder tumor markers beyond cytology: International Consensus Panel on bladder tumor markers. Urology 66 (6 Suppl 1): S35-S63, 2005.

8. van Rhijn BW, van der Poel HG and van der Kwast TH: Urine markers for bladder cancer surveillance: A systematic review. Eur Urol 47: 736-748, 2005

9. Vrooman OP and Witjes JA: Urinary markers in bladder cancer. Eur Urol 53: 909-916, 2008.

10. Xylinas E, Kluth LA, Rieken M, Karakiewicz PI, Lotan Y and Shariat SF: Urine markers for detection and surveillance of bladder cancer. Urol Oncol 32: 222-229, 2014.

11. Smith ZL and Guzzo TJ: Urinary markers for bladder cancer. F1000Prime Reports 5: 21, 2013.

12. Sakamoto K and Kadomatsu K: Midkine in the pathology of cancer, neural disease, and inflammation. Pathol Int 62: 445-455, 2012.

13. Jones DR: Measuring midkine: The utility of midkine as a biomarker in cancer and other diseases. Br J Pharmacol 171: 2925-2939, 2014.

14. Velculescu VE, Madden SL, Zhang L, Lash AE, Yu J, Rago C, Lal A, Wang CJ, Beaudry GA, Ciriello KM, et al: Analysis of human transcriptomes. Nat Genet 23: 387-388, 1999.

15. Ikematsu S, Okamoto K, Yoshida Y, Oda M, Sugano-Nagano H, Ashida K, Kumai H, Kadomatsu K, Muramatsu H, Muramatsu T and Sakuma S: High levels of urinary midkine in various cancer patients. Biochem Biophys Res Commun 306: 329-332, 2003.

16. O'Brien T, Cranston D, Fuggle S, Bicknell R and Harris AL: The angiogenic factor midkine is expressed in bladder cancer, and overexpression correlates with a poor outcome in patients with invasive cancers. Cancer Res 56: 2515-2518, 1996.

17. Shimwell NJ, Bryan RT, Wei W, James ND, Cheng KK, Zeegers MP, Johnson PJ, Martin A and Ward DG: Combined proteome and transcriptome analyses for the discovery of urinary biomarkers for urothelial carcinoma. Br J Cancer 108: 1854-1861, 2013.

18. Soukup V, Kalousová M, Capoun O, Sobotka R, Breyl Z, Pešl M, Zima T and Hanuš T: Panel of urinary diagnostic markers for non-invasive detection of primary and recurrent urothelial urinary bladder carcinoma. Urol Int 95: 56-64, 2015.

19. Sobin LH, Gospodarowicz M and Wittekind C (eds): TNM Classification of Malignant Tumors. 7th edition. Wiley-Blackwell, Hoboken, NJ, pp262-265, 2009.

20. Eble JN, Sauter G, Epstein JI and Sesterhenn IA (eds): World Health Organization Classification of Tumours. Pathology and Genetics of Tumours of the Urinary System and Male Genital Organs IARC Press, Lyon, pp87-157, 2004.

21. Mostofi FK, Sobin LH and Torlonie H: Histological Typing of Urinary Bladder Tumours. Vol 10. World Health Organization, Geneva, pp17, 1973.

22. Bossuyt PM, Reitsma JB, Bruns DE, Gatsonis CA, Glasziou PP, Irwig LM, Moher D, Rennie D, de Vet HC and Lijmer JG; Standards for Reporting of Diagnostic Accuracy Group: The STARD statement for reporting studies of diagnostic accuracy: Explanation and elaboration. The Standards for Reporting of Diagnostic Accuracy Group. Croat Med J 44: 639-650, 2003.

23. Papanicolaou GN and Marshall VF: Urine sediment smears as a diagnostic procedure in cancers of the urinary tract. Science 101: $519-520,1945$.

24. Youden WJ: Index for rating diagnostic tests. Cancer 3: 32-35, 1950.

25. Lalkhen AG and McCluskey A: Clinical tests: Sensitivity and specificity. Contin Educ Anaesth Crit Care Pain 8: 221-223, 2008.

26. Todenhöfer T, Hennenlotter J, Witstruk M, Gakis G, Aufderklamm S, Kuehs U, Stenzl A and Schwentner C: Influence of renal excretory function on the performance of urine based markers to detect bladder cancer. J Urol 187: 68-73, 2012.

27. Konishi N, Nakamura M, Nakaoka S, Hiasa Y, Cho M, Uemura H, Hirao Y, Muramatsu T and Kadomatsu K: Immunohistochemical analysis of midkine expression in human prostate carcinoma. Oncology 57: 253-257, 1999.

28. Davies B, Chen J, Modugno F, Weissfeld J, Landsittel D, Dhir R, Nelson J and Getzenberg RH: Contribution of the prostate limits the usefulness of survivin for the detection of bladder cancer. J Urol 174: 1767-1770, 2005.

29. Boman H, Hedelin $\mathrm{H}$ and Holmäng S: Four bladder tumor markers have a disappointingly low sensitivity for small size and low grade recurrence. J Urol 167: 80-83, 2002. 
30. Lotan Y and Roehrborn CG: Sensitivity and specificity of commonly available bladder tumor markers versus cytology: Results of a comprehensive literature review and meta-analyses. Urology 61: 109-118, 2003.

31. Sun Y, He DL, Ma Q, Wan XY, Zhu GD, Li L, Luo Y, He H and Yang L: Comparison of seven screening methods in the diagnosis of bladder cancer. Chin Med J (Engl) 119: 1763-1771, 2006.

32. Holyoake A, O'Sullivan P, Pollock R, Best T, Watanabe J, Kajita Y, Matsui Y, Ito M, Nishiyama H, Kerr N, et al: Development of a multiplex RNA urine test for the detection and stratification of transitional cell carcinoma of the bladder. Clin Cancer Res 14: 742-749, 2008.

33. Ikematsu S, Nakagawara A, Nakamura Y, Sakuma S, Wakai K, Muramatsu T and Kadomatsu K: Correlation of elevated level of blood midkine with poor prognostic factors of human neuroblastomas. Br J Cancer 88: 1522-1526, 2003.

34. Sánchez-Carbayo M, Urrutia M, González de Buitrago JM and Navajo JA: Evaluation of two new urinary tumor markers: Bladder tumor fibronectin and cytokeratin 18 for the diagnosis of bladder cancer. Clin Cancer Res 6: 3585-3594, 2000.

35. Abd El-Hakim TF, El-Shafie MK, Abdou AG, Azmy RM, El-Naidany SS and Badr El-Din MO: Value of urinary survivin as a diagnostic marker in bladder cancer. Anal Quant Cytopathol Histpathol 36: 121-127, 2014.

36. Konety BR: Molecular markers in bladder cancer: A critical appraisal. Urol Oncol 24: 326-337, 2006.

37. Yafi FA, Brimo F, Steinberg J, Aprikian AG, Tanguay S and Kassouf W: Prospective analysis of sensitivity and specificity of urinary cytology and other urinary biomarkers for bladder cancer. Urol Oncol 33: e25-e31, 2015.

38. Ye F, Wang L, Castillo-Martin M, McBride R, Galsky MD, Zhu J, Boffetta P,Zhang DY and Cordon-Cardo C: Biomarkers for bladder cancer management: Present and future. Am J Clin Exp Urol 2: $1-14,2014$.

39. Raab SS, Grzybicki DM, Vrbin CM and Geisinger KR: Urine cytology discrepancies-Frequency, causes, and outcomes. Am J Clin Pathol 127: 946-953, 2007.

40. Reid MD, Osunkoya AO, Siddiqui MT and Looney SW: Accuracy of grading of urothelial carcinoma on urine cytology: An analysis of interobserver and intraobserver agreement. Int J Clin Exp Pathol 5: 882-891, 2012.

41. Sullivan PS, Chan JB, Levin MR and Rao JY: Urine cytology and adjunct markers for detection and surveillance of bladder cancer. Am J Transl Res 2: 412-440, 2010.
42. Ku JH, Godoy G, Amiel GE and Lerner SP: Urine survivin as a diagnostic biomarker for bladder cancer: A systematic review. BJU Int 110: 630-636, 2012.

43. Schultz IJ, Witjes JA, Swinkels DW and de Kok JB: Bladder cancer diagnosis and recurrence prognosis: Comparison of markers with emphasis on survivin. Clin Chim Acta 368: 20-32, 2006

44. Srivastava AK, Singh PK, Srivastava K, Singh D, Dalela D, Rath SK, Goel MM and Brahma Bhatt ML: Diagnostic role of survivin in urinary bladder cancer. Asian Pac J Cancer Prev 14: 81-85, 2013.

45. Vriesema JL, Poucki MH, Kiemeney LA and Witjes JA: Patient opinion of urinary tests versus flexible urethrocystoscopy in follow-up examination for superficial bladder cancer: A utility analysis. Urology 56: 793-797, 2000.

46. Yossepowitch O, Herr HW and Donat SM: Use of urinary biomarkers for bladder cancer surveillance: Patient perspectives. J Urol 177: 1277-1282, 2007.

47. Todenhöfer T, Hennenlotter J, Esser M, Mohrhardt S, Tews V, Aufderklamm S, Gakis G, Kuehs U, Stenzl A and Schwentner C: Combined application of cytology and molecular urine markers to improve the detection of urothelial carcinoma. Cancer cytopathology 121: 252-260, 2013

48. Todenhöfer T, Hennenlotter J, Guttenberg P, Mohrhardt S, Kuehs U, Esser M, Aufderklamm S, Bier S, Harland N, Rausch S, et al: Prognostic relevance of positive urine markers in patients with negative cystoscopy during surveillance of bladder cancer. BMC Cancer 15: 155, 2015.

49. Eissa S, Salem AM, Zohny SF and Hegazy MG: The diagnostic efficacy of urinary TGF-beta1 and VEGF in bladder cancer: Comparison with voided urine cytology. Cancer Biomark 3: 275-285, 2007.

50. Lokeshwar VB, Obek C, Pham HT, Wei D, Young MJ, Duncan RC, Soloway MS and Block NL: Urinary hyaluronic acid and hyaluronidase: Markers for bladder cancer detection and evaluation of grade. J Urol 163: 348-356, 2000.

51. Schmidt J, Propping C, Siow WY, Lohse-Fischer A, Toma M, Baldauf-Twelker A, Hakenberg OW, Wirth MP and Fuessel S: Diagnostic and prognostic value of bladder cancer-related transcript markers in urine. J Cancer Res Clin Oncol 142: 401-414, 2016. 